PROCEEDINGS OF THE

AMERICAN MATHEMATICAL SOCIETY

Volume 136, Number 6, June 2008, Pages 2147-2153

S 0002-9939(08)09197-1

Article electronically published on February 14, 2008

\title{
ITERATING THE CESÀRO OPERATORS
}

\author{
FERNANDO GALAZ FONTES AND FRANCISCO JAVIER SOLÍS
}

(Communicated by Joseph A. Ball)

\begin{abstract}
The discrete Cesàro operator $C$ associates to a given complex sequence $s=\left\{s_{n}\right\}$ the sequence $C s \equiv\left\{b_{n}\right\}$, where $b_{n}=\frac{s_{0}+\cdots+s_{n}}{n+1}, n=$ $0,1, \ldots$ When $s$ is a convergent sequence we show that $\left\{C^{n} s\right\}$ converges under the sup-norm if, and only if, $s_{0}=\lim _{n \rightarrow \infty} s_{n}$. For its adjoint operator $C^{*}$, we establish that $\left\{\left(C^{*}\right)^{n} s\right\}$ converges for any $s \in \ell^{1}$.

The continuous Cesàro operator, $C f(x) \equiv \frac{1}{x} \int_{0}^{x} f(s) d s$, has two versions: the finite range case is defined for $f \in L^{\infty}(0,1)$ and the infinite range case for $f \in L^{\infty}(0, \infty)$. In the first situation, when $f:[0,1] \rightarrow \mathbb{C}$ is continuous we prove that $\left\{C^{n} f\right\}$ converges under the sup-norm to the constant function $f(0)$. In the second situation, when $f:[0, \infty) \rightarrow \mathbb{C}$ is a continuous function having a limit at infinity, we prove that $\left\{C^{n} f\right\}$ converges under the sup-norm if, and only if, $f(0)=\lim _{x \rightarrow \infty} f(x)$.
\end{abstract}

\section{INTRODUCTION}

We will denote by $\mathcal{S}$ the vector space consisting of all complex sequences. If $s \in \mathcal{S}$, we will write $s=\left\{s_{n}: n \in \mathbb{N}_{0}\right\}$ or $s=\left\{s(n): n \in \mathbb{N}_{0}\right\}$, where $\mathbb{N}_{0} \equiv\{0\} \cup \mathbb{N}$. Given $s \in \mathcal{S}$, let $b$ be the sequence given by

$$
b_{n} \equiv \frac{s_{0}+\ldots+s_{n}}{n+1}, n \in \mathbb{N}_{0} .
$$

Then $C: \mathcal{S} \rightarrow \mathcal{S}$ defined by $C s \equiv b$ is the (discrete) Cesàro operator.

As usual, let $c$ be the Banach space consisting of all convergent sequences together with the sup-norm $\|\cdot\|_{\infty}$, and $c_{0}$ be its (closed) subspace formed by those sequences converging to 0 . We will denote by $e_{k}$ the sequence satisfying $e_{k}(m)=\delta_{k, m}, k, m \in$ $\mathbb{N}_{0}$. The following two linear functionals defined on $c$ will play a key role:

$$
L s \equiv \lim _{n \rightarrow \infty} s_{n}, \quad \pi(s) \equiv s(0) .
$$

Clearly each of them is bounded. It is well known that $C(c) \subset c, C\left(c_{0}\right) \subset c_{0}$ and $L C s=L s, \forall s \in c$. We also have $\pi C s=\pi s, \forall s \in c$. Moreover, for $X=c, c_{0}$, the operator $C: X \rightarrow X$ is bounded and $\|C\|=1$.

It is well known that $C s$ may converge, although the bounded sequence $s$ does not converge. So in the sense of convergence, we may think of this fact as $C$ making "better" sequences. Thus the question arises as to how does the sequence of iterates

Received by the editors November 10, 2006 and, in revised form, April 12, 2007.

2000 Mathematics Subject Classification. Primary 47B37, 40 G05.

Key words and phrases. Cesàro operator, Iterates, Hausdorff operator.

The first author was partially supported by CONACyT (México) project 49187-F.

The second author was partially supported by CONACyT (México) project 50926-F.

(C) 2008 American Mathematical Society Reverts to public domain 28 years from publication 
$\left\{C^{n} s\right\}$ behave? For $s \in c$, in Theorem 1 we prove that $\left\{C^{n} s\right\}$ converges if, and only if, $s(0)=L s$. In this case we have that $\left\{C^{n} s\right\}$ converges to the constant sequence $s(0)$.

Theorem 2 deals with the iterates of $C^{*}$, the adjoint operator for $C: c_{0} \rightarrow c_{0}$. We show that for any $y \in \ell^{1}$ the sequence $\left\{\left(C^{*}\right)^{n} y\right\}$ converges to $\varphi(y) e_{0}$ where $\varphi(y)=\sum_{j=0}^{\infty} y(j)$.

We also analyze the finite range and the infinite range cases for the continuous Cesàro operator. (One can find in 4] a very interesting exposition of the main properties of the Cesàro operators.)

In the finite range case we consider $f \in L^{\infty}(0,1)$ and define

$$
C f(x) \equiv \frac{1}{x} \int_{0}^{x} f(s) d s, \forall x \in(0,1)
$$

Then $C f \in L^{\infty}(0,1)$ and we obtain a linear operator $C: L^{\infty}(0,1) \rightarrow L^{\infty}(0,1)$ with $\|C\|=1$. For $f \in C[0,1]$ we extend the above definition by taking

$$
C f(0) \equiv f(0), \quad C f(1)=\int_{0}^{1} f(s) d s .
$$

In this situation we also have that $C: C[0,1] \rightarrow C[0,1]$ is a bounded linear operator and $\|C\|=1$. For $f \in C[0,1]$, we show in Theorem 3 that $\left\{C^{n} f\right\}$ always converges to the constant function $f(0)$.

In the infinite range case we consider $f \in L^{\infty}(0, \infty)$ and define

$$
C f(x) \equiv \frac{1}{x} \int_{0}^{x} f(s) d s, \forall x \in(0, \infty) .
$$

Then $C f \in L^{\infty}(0, \infty)$ and we obtain a linear operator $C: L^{\infty}(0, \infty) \rightarrow L^{\infty}(0, \infty)$ with $\|C\|=1$. If $f \in C[0, \infty)$ is bounded take $C f(0) \equiv f(0)$ and let us denote by $C[0, \infty]$ the closed subspace of $L^{\infty}(0, \infty)$ consisting of all continuous functions $f:[0, \infty) \rightarrow \mathbb{C}$ such that $\lim _{x \rightarrow \infty} f(x)$ exists. In this situation we also have that $C$ takes $C[0, \infty]$ into itself and $C: C[0, \infty] \rightarrow C[0, \infty]$ is a bounded linear operator with $\|C\|=1$. For $f \in C[0, \infty]$, we prove in Theorem 4 that $\left\{C^{n} f\right\}$ converges if, and only if, $f(0)=\lim _{x \rightarrow \infty} f(x)$. In this case we have that $\left\{C^{n} f\right\}$ converges to the constant function $f(0)$, a result corresponding to that of the discrete case.

\section{Discrete CASE}

We will start by discussing a finite dimensional case for the Cesàro operator that throws light on the general situation. So let $m=0,1, \ldots$ and consider $\mathbb{C}^{m+1}$ with the sup-norm $\left\|\left(b_{0}, \ldots, b_{m}\right)\right\| \equiv \max \left\{\left|b_{0}\right|, \ldots,\left|b_{m}\right|\right\}$. The Cesàro operator now takes the form

$$
C\left(s_{0}, s_{1}, \ldots, s_{m}\right) \equiv\left(s_{0}, \frac{s_{0}+s_{1}}{2}, \ldots, \frac{s_{0}+s_{1}+\cdots+s_{m}}{m+1}\right) .
$$

For $s=\left(s_{0}, \ldots, s_{m}\right) \in \mathbb{C}^{m+1}$, let $M \equiv\left\{\left(s_{0}, x_{1}, \ldots, x_{m}\right): x_{1}, \ldots, x_{m} \in \mathbb{K}\right\}$. Notice $s \in M$ and $C(M) \subset M$. Take $x=\left(s_{0}, x_{1}, \ldots, x_{m}\right)$ and $y=\left(s_{0}, y_{1}, \ldots, y_{m}\right) \in M$. Then,

$$
\left\|\frac{s_{0}+x_{1}+\cdots+x_{j}}{j+1}-\frac{s_{0}+y_{1}+\cdots+y_{j}}{j+1}\right\| \leq \frac{j}{j+1}\|x-y\|, 2 \leq j \leq m .
$$

It follows that

$$
\|C x-C y\| \leq K\|x-y\|, \forall y \in M,
$$


with $K \equiv\left(1-\frac{1}{m+1}\right)<1$. This shows that $C$ is a contraction on $M$ and so it has a unique fixed point, which is easily seen to be the constant vector $\left(s_{0}, \ldots, s_{0}\right)$. Thus we have proved the following.

Proposition 1. If $s=\left(s_{0}, s_{1}, \ldots, s_{m}\right) \in \mathbb{C}^{m+1}$, then $C^{n} s \rightarrow\left(s_{0}, \ldots, s_{0}\right)$.

We now consider the infinite dimensional case.

Theorem 1. Let $s \in c$. Then $\left\{C^{n} s\right\}$ converges if, and only if, $s_{0}=L(s)$. In this case, $\left\{C^{n} s\right\}$ converges to (the constant sequence) $s_{0}$.

Proof. Assume $C^{n} s \rightarrow y$. This implies $s(0)=\pi\left(C^{n}(s)\right) \rightarrow \pi(y)=y(0)$, when $n \rightarrow \infty$. Thus $s(0)=y(0)$. We also have $L s=L\left(C^{n} s\right) \rightarrow L y$, when $n \rightarrow \infty$. It follows that $L y=L s$. From $C^{n} s \rightarrow y$ we have that $y$ is constant and so $L y=y(0)$. Hence $s(0)=L s$.

To establish the other implication, we will first prove

$$
C^{n}\left(e_{k}\right) \rightarrow 0 \text { when } n \rightarrow \infty, \forall k=1,2, \ldots
$$

Let us fix $n \in \mathbb{N}$. According to G. H. Hardy [2, Sect. 11.12], $C^{n}$ is the moment difference operator corresponding to the measure on the interval $[0,1]$ given by $d \mu \equiv f_{n}(t) d t$, where

$$
f_{n}(t) \equiv \frac{1}{(n-1) !} \log ^{n-1} \frac{1}{t}, 0<t \leq 1 .
$$

(A brief discussion of this result can be found in [3, p. 125].) This means that for any $s \in c$ we have

$$
C^{n} s(m) \equiv \sum_{j=0}^{m}\left(\begin{array}{c}
m \\
j
\end{array}\right) s_{j} \int_{0}^{1}(1-t)^{m-j} t^{j} f_{n}(t) d t, \forall m \in \mathbb{N}_{0} .
$$

Now take $k \in \mathbb{N}$. From above we have $C^{n} e_{k}(m)=0, m<k$, and

$$
C^{n} e_{k}(m)=\left(\begin{array}{c}
m \\
k
\end{array}\right) \int_{0}^{1}(1-t)^{m-k} t^{k} f_{n}(t) d t, k \leq m .
$$

Let us define $g_{n}(0)=0, g_{n}(t)=t f_{n}(t), 0<t \leq 1$ and

$$
a_{n} \equiv \sup \left\{g_{n}(t): 0 \leq t \leq 1\right\} .
$$

Since $\int_{0}^{1}(1-t)^{m-k} t^{k-1} d t=\frac{(m-k) !(k-1) !}{m !}$ [5, Thm. 7.69], from (66) we obtain $\left|C^{n} e_{k}(m)\right| \leq \frac{a_{n}}{k}$. Thus

$$
\left\|C^{n} e_{k}\right\|_{\infty} \leq a_{n}, \forall k \in \mathbb{N} .
$$

Assume in what follows that

$$
a_{n} \rightarrow 0
$$

Then (4) is obtained from (8) and (9).

Take $s \in c_{0}$ such that $s(0)=0$ and let $\sigma_{N} \equiv \sum_{k=0}^{N} s_{k} e_{k} \equiv \sum_{k=1}^{N} s_{k} e_{k}$. Given $\epsilon>0$, we have $\left\|s-\sigma_{N_{1}}\right\|_{\infty} \leq \frac{\epsilon}{2}$ for some $N_{1} \in \mathbb{N}$. From (41), we can find some $N>N_{1}$ such that $\left\|C^{n} \sigma_{N}\right\|_{\infty} \leq \frac{\epsilon}{2} \forall n \geq N$. Hence

$$
\left\|C^{n} s\right\|_{\infty} \leq\left\|C^{n} \sigma_{N}\right\|_{\infty}+\left\|C^{n}\left(s-\sigma_{N}\right)\right\|_{\infty} \leq \frac{\epsilon}{2}+\left\|s-\sigma_{N}\right\|_{\infty} \leq \epsilon, \forall n \geq N .
$$


Finally, let $s \in c$ be such that $s(0)=L s$. Then, $s=(s-s(0))+s(0)$. Since $s-s_{0} \in c_{0}$ and $\pi(s-s(0))=0$, we have

$$
C^{n} s=C^{n}\left(s-s_{0}\right)+C^{n} s_{0}=C^{n}\left(s-s_{0}\right)+s_{0} \rightarrow s_{0} .
$$

All that is now left to prove is (9), and we do this in the following lemma.

Lemma 1. $a_{n} \rightarrow 0$ when $n \rightarrow \infty$.

Proof. Fix $n \in \mathbb{N}, n \geq 2$, and notice that $g_{n}:[0,1] \rightarrow \mathbb{R}$ is continuous. Its derivative is

$$
g_{n}^{\prime}(t)=\frac{\ln ^{n-2} \frac{1}{t}}{(n-2) !}\left(\frac{\ln \frac{1}{t}}{n-1}-1\right), 0<t \leq 1 .
$$

After simple calculations it follows that $g_{n}$ has $t_{0} \equiv e^{-n+1}$ as its unique critical point and that $g_{n}\left(t_{0}\right)$ is its maximum value. Thus

$$
a_{n}=g_{n}\left(t_{0}\right)=\frac{e^{-n+1}(n-1)^{n-1}}{(n-1) !} .
$$

Stirling's formula states that $\lim _{m \rightarrow \infty}\left[\frac{m !}{\left.e^{-m} m^{m} \sqrt{2 \pi m}\right)}\right]=1$ [5, Thm. 5.44]. From this and (10) the conclusion follows.

\section{Iterates of the ADJoint of the Cesìro operator}

The next result extends Proposition 1 to $\ell^{\infty}$ and complements Theorem 1. Since $\ell^{\infty}=\left(\ell^{1}\right)^{*}$, notice that $\ell^{\infty}$ can be given the weak-* topology.

Corollary 1. $\left\{C^{n} s\right\}$ converges weak-* to (the constant sequence) $s_{0}$, for any $s \in$ $\ell^{\infty}$.

Proof. Consider $s \in \ell^{\infty}, s \neq 0$. Take $y \in \ell^{1}$ and let $\epsilon>0$ be given. First we fix $N \in \mathbb{N}$ to satisfy $\left\|y-y_{N}\right\| \leq \frac{\epsilon}{4\|s\|_{\infty}}$, where $y_{N} \equiv \sum_{j=0}^{N} y(j) e_{j}$. Since $\left\|C^{n}\right\| \leq 1$, this implies

$$
\begin{aligned}
\left|\left\langle C^{n} s-s_{0}, y\right\rangle\right| & =\left|\left\langle C^{n} s-s_{0}, y_{N}\right\rangle\right|+\left|\left\langle C^{n} s-s_{0}, y-y_{N}\right\rangle\right| \\
& \leq \sum_{j=0}^{N}\left(C^{n} s(j)-s_{0}\right) y(j)+\frac{\epsilon}{2} .
\end{aligned}
$$

From Proposition 1 follows that, for each $j \in \mathbb{N}_{0}, C^{n} s(j) \rightarrow s_{0}$ when $n \rightarrow \infty$. Using this in (11), we conclude that $\left\langle C^{n} s, y\right\rangle \rightarrow\left\langle s_{0}, y\right\rangle$.

We now consider $C: c_{0} \rightarrow c_{0}$. After some simple calculations we find that its adjoint $C^{*}: \ell^{1} \rightarrow \ell^{1}$ is given by

$$
C^{*} y(m)=\sum_{j=m}^{\infty} \frac{y(j)}{j+1}, m \in \mathbb{N}_{0} .
$$

Theorem 2. $\left(C^{*}\right)^{n} y \rightarrow\left(\sum_{j=0}^{\infty} y(j)\right) e_{0}, \forall y \in \ell^{1}$. 
Proof. Let $y \in \ell^{1}$. Since weakly convergent sequences in $\ell^{1}$ are norm convergent, to obtain the conclusion we only have to show that $\left.\left\{C^{*}\right)^{n} y\right\}$ converges weakly to $\left(\sum_{j=0}^{\infty} y(j)\right) e_{0}$. Take $s \in \ell^{\infty}=\ell^{1^{*}}$. From Corollary 1 we now obtain

$$
\begin{aligned}
\left\langle\left(C^{*}\right)^{n} y, s\right\rangle & =\left\langle y, C^{n} s\right\rangle \rightarrow\left\langle y, s_{0}\right\rangle=\sum_{j=0}^{\infty} y(j) s_{0} \\
& =\left\langle\left(\sum_{j=0}^{\infty} y(j)\right) e_{0}, s\right\rangle .
\end{aligned}
$$

\section{THE FINITE RANGE CASE}

Next we will see that the behavior of the Cesàro operator on the space of continuous complex functions $C[0,1]$ is the same as that of the Cesàro operator defined on $\mathbb{C}^{n}$.

Theorem 3. $C^{n} f \rightarrow f(0), \forall f \in C[0,1]$.

Proof. By a direct calculation we obtain

$$
C x^{k}=\frac{1}{k+1} x^{k}, k \in \mathbb{N}_{0} .
$$

Thus $C^{n} 1=1$ and $C^{n} x^{k} \rightarrow 0, \forall k \in \mathbb{N}$. Let $P(x) \equiv c_{0}+c_{1} x+\cdots+c_{m} x^{m}$ be a polynomial. Hence

$$
C^{n} P \equiv c_{0}+\frac{1}{2^{n}} c_{1} x+\cdots+\frac{1}{(m+1)^{n}} c_{m} x^{m} .
$$

It follows that $C^{n} P \rightarrow c_{0}=P(0)$.

We now consider an arbitrary function $f \in C[0,1]$ and let a positive real number $\epsilon$ be given. Applying Weierstrass' Theorem we find a polynomial $P$ such that $\|f-P\| \leq \frac{\epsilon}{3}$. By the case discussed above, there is some $N \in \mathbb{N}$ such that $\left\|C^{n} P-P(0)\right\| \leq \frac{\epsilon}{3}, \forall n \geq N$. Let $n \geq N$. Since $\left\|C^{n}\right\| \leq 1$, it follows that

$$
\begin{aligned}
\left\|C^{n} f-f(0)\right\| & =\left\|C^{n} f-C^{n} f(0)\right\| \\
& \leq\left\|\left(C^{n} f-C^{n} P\right)\right\|+\left\|\left(C^{n}\left(P-C^{n} f(0)\right)\right)\right\| \\
& =\|(f-P)\|+\left\|C^{n} P-P(0)\right\|+\|P(0)-f(0)\| \leq \epsilon .
\end{aligned}
$$

\section{The infinite RANGe CASE}

Recall that $C[0, \infty]$ consists of all continuous functions $f:[0, \infty) \rightarrow \mathbb{C}$ such that $\lim _{x \rightarrow \infty} f(x)$ exists. To analyze $C:[0, \infty] \rightarrow[0, \infty]$ we will proceed in a way similar to that of the discrete Cesàro operator. We define

$$
L f \equiv \lim _{x \rightarrow \infty} f(x), \quad \pi(f) \equiv f(0), \forall f \in C[0, \infty] .
$$

Clearly both $L$ and $\pi$ are bounded linear functionals. Moreover, they satisfy

$$
L C f=L f, \quad \pi C f=\pi f, \forall f \in C[0, \infty] .
$$

Using the change of variables $s=x t$, (3) can be written as

$$
C f(x)=\int_{0}^{1} f(x t) d t, \forall x \in(0, \infty) .
$$


More generally, D. W. Boyd proved that

$$
C^{n} f(x)=\int_{0}^{1} f(x t) f_{n}(t) d t, \forall x \in(0, \infty)
$$

where $f_{n}$ is given by (5) [1, Lemma 2].

Theorem 4. Let $f \in C[0, \infty]$. Then $\left\{C^{n} f\right\}$ converges if, and only if, $f(0)=L f$. In this case, $\left\{C^{n} f\right\}$ converges to (the constant function) $f(0)$.

Proof. The necessity of the condition is established as in Theorem 1.

To establish sufficiency, we will first assume $f(0)=0=L f$. Let $\epsilon>0$ be given. We can now choose $\delta$ such that $0<\delta<1$ and $N \in \mathbb{N}$ to satisfy

$$
|f(u)| \leq \frac{\epsilon}{3} \text { if } 0 \leq u \leq \delta \text { or } u \geq N .
$$

Let $x>N$. To estimate $C^{n} f(x)$ using (13), we divide the integration interval $[0,1]$ in three parts. Since $\int_{0}^{1} f_{n}(t) d t=1$, we have

$$
\int_{0}^{\frac{\delta}{x}}|f(x t)| f_{n}(t) d t \leq \frac{\epsilon}{3} \int_{0}^{1} f_{n}(t) d t=\frac{\epsilon}{3} .
$$

Similarly, we obtain

$$
\int_{\frac{N}{x}}^{1}|f(x t)| f_{n}(t) d t \leq \frac{\epsilon}{3} \int_{0}^{1} f_{n}(t) d t=\frac{\epsilon}{3} .
$$

Next, using that $f_{n}$ is a decreasing function and (7), we find

$$
\int_{\frac{\delta}{x}}^{\frac{N}{x}}|f(x t)| f_{n}(t) d t \leq\|f\|_{\infty} \frac{(N-\delta)}{x} f_{n}\left(\frac{\delta}{x}\right) \leq\|f\|_{\infty} \frac{(N-\delta)}{\delta} a_{n} .
$$

Applying Lemma 1 this implies

$$
\int_{\frac{\delta}{x}}^{\frac{N}{x}}|f(x t)| f_{n}(t) d t \leq \frac{\epsilon}{3}, \forall n \geq N_{1},
$$

for some $N_{1} \in \mathbb{N}$.

Finally, by (13), (14), (15) and (16) we conclude that

$$
\left|C^{n} f(x)\right| \leq \epsilon, \forall x>N, \forall n \geq N_{1} .
$$

Now, from the finite range case (with the interval $[0, N]$ instead of $[0,1]$ ) we find $N_{2} \in \mathbb{N}$ such that

$$
\left|C^{n} f(x)\right| \leq \epsilon, \forall x \in[0, N], \forall n \geq N_{2} .
$$

This proves the theorem when $f \in C[0, \infty]$ satisfies $f(0)=L f=0$. If $f(0)=L f$, then we proceed as in the discrete case.

\section{ACKNOWLEDGEMENT}

The authors gratefully acknowledge the referee for pointing out the role of weak-* convergence in our work. He established Corollary 1 and considerably simplified our original proof for Theorem 2 . 


\section{REFERENCES}

[1] D. W. Boyd, 'The spectral radius of averaging operators'. Pacific J. Math., 24(1968), No. 1, 19-28. MR0221308 (36:4360)

[2] G. H. Hardy, Divergent series. Clarendon Press, Oxford, 1949. MR.0030620 (11:25a)

[3] G. Leibowitz, 'Spectra of discrete Cesàro operators'. Tamkang J. Math., 3(1972), 123-132. MR0318948 (47:7494)

[4] G. Leibowitz, 'The Cesàro operators and their generalizations: examples in infinite dimensional linear analysis'. Amer. Math. Monthly, 80(6)(1973), 654-661. MR0322569 (48:931)

[5] K. Stromberg, An introduction to classical real analysis. Wadsworth International Group, Belmont, California, 1981. MR604364 (82c:26002)

Uam-Iztapalapa, Av. San Rafael Atlixco 186, Col. Vicentina, México D. F., C. P. 09340

E-mail address: galaz@cimat.mx

Cimat, Apdo. Postal 402, 36000 Guanajuato, Gto., Mexico

E-mail address: solis@cimat.mx 